\title{
A role for BELLRINGER in cell wall development is supported by loss-of-function phenotypes
}

\author{
J Peter Etchells ${ }^{1,3^{*}+}$, Lucy Moore ${ }^{1 \dagger}$, Wen Zhi Jiang ${ }^{1,4}$, Helen Prescott ${ }^{1}$, Richard Capper ${ }^{1,5}$, Nigel J Saunders ${ }^{2,6}$, \\ Anuj M Bhatt ${ }^{1}$ and Hugh G Dickinson ${ }^{1^{*}}$
}

\begin{abstract}
Background: Homeodomain transcription factors play critical roles in metazoan development. BELLRINGER (BLR), one such transcription factor, is involved in diverse developmental processes in Arabidopsis, acting in vascular differentiation, phyllotaxy, flower and fruit development. $B L R$ also has a redundant role in meristem maintenance. Cell wall remodelling underpins many of these processes, and $B L R$ has recently been shown to regulate expression of PECTIN METHYL-ESTERASE 5 (PME5), a cell wall modifying enzyme in control of phyllotaxy. We have further explored the role of $B L R$ in plant development by analysing phenotypes and gene expression in a series of plants over-expressing $B L R$, and generating combinatorial mutants with blr, brevipedicellus (bp), a member of the KNOX1 family of transcription factors that has previously been shown to interact with blr, and the homeodomain transcription factor revoluta (rev), required for radial patterning of the stem.

Results: Plants over-expressing BLR exhibited a wide range of phenotypes. Some were defective in cell size and demonstrated misregulation of genes predominantly affecting cell wall development. Other lines with more extreme phenotypes failed to generate lateral organs, consistent with BLR repressing transcription in the shoot apex. Cell wall dynamics are also affected in blr mutant plants, and $B L R$ has previously been shown to regulate vascular development in conjunction with BP. We found that when $b p$ and blr were combined with rev, a set of defects was observed that were distinct from those of bp blr lines. In these triple mutants xylem development was most strikingly affected, resulting in an almost complete lack of vessels and xylem parenchyma with secondary thickening.

Conclusions: Our data support a role for BLR in ordering the shoot apex and, in conjunction with BP and REV, playing a part in determining the composition and organisation of the vascular system. Microarray analysis strongly indicates that the striking vascular phenotypes of blr bp rev triple mutants and plants over-expressing $B L R$ result from the misregulation of a suite of genes, targets of BLR in wild type plants, that determine cell size and structure in the developing vasculature.
\end{abstract}

\section{Background}

BELLRINGER (BLR), also known as VAAMANA, PENNYWISE, LARSON and REPLUMLESS, is a member of the $B E L L$ family of homeodomain transcription factors and functions in diverse processes in the development of Arabidopsis [1-5]. BLR was identified independently as a

\footnotetext{
*Correspondence: Peter.Etchells@manchester.ac.uk; Hugh.Dickinson@plants. ox.ac.uk

${ }^{\dagger}$ Equal contributors

'Department of Plant Sciences, University of Oxford, South Parks Road, Oxford, UK

${ }^{3}$ Faculty of Life Sciences, University of Manchester, Manchester, UK Full list of author information is available at the end of the article
}

suppresser of the floral homeotic gene AGAMOUS (AG) [5], as a factor required in fruit development for specification of the replum [1] and as a gene necessary for normal phyllotactic patterning $[2,4]$. BELL transcription factors were shown to enhance DNA binding of maize KNOTTED1 [6], the founding member of the KNOX1 gene family [7], and $B L R$ was subsequently shown to act synergistically with the KNOX1 homeodomain transcription factor BREVIPEDICELLUS (BP) [3]. BLR and KNOX1 proteins heterodimerize [3] and, in Nicotiana epidermal cell transient assays, BLR translocates to the nucleus on binding to KNOX1 proteins [2]. This event is

\section{Biomed Central}


likely to have evolved early in eukaryotic development as it is required for the haploid-diploid transition in the green algae Chlamydomonas [8].

Although the expression patterns of $B L R$ and $K N O X 1$ overlap, they are not identical [3] and blr and knox1 mutants show distinctive phenotypes, suggesting that BLR function is unlikely to be confined to acting with KNOX1 proteins alone. Nevertheless, similarities are apparent, such as the short stature of both $b l r$ and $b p$ plants [2-4] and the ability of $b l r$ and $b p$ to enhance weak alleles of shoot meristemless (stm) [2,4], a KNOX1 gene required for shoot apical meristem maintenance $[9,10]$, which points to a redundant role for $B L R$ and $B P$ in stem cell fate establishment [2-4]. Reports of genetic interactions between $b l r$ and $b p$ mutants have, however, been conflicting, with the short stature phenotype of the bp blr double mutation being variously interpreted as additive [4], synergistic [3], or novel - because inflorescence elongation is observed in the single mutants but not in the double [2]. Phenotypic differences between $b l r$ and $b p$ mutants are unlikely to result from redundancy between family members because multiple knockout lines within the KNOX1 and BELL families exhibit diverse phenotypes. For example, vegetative to reproductive transition defects are observed when blr mutants are combined with mutations in poundfoolish (pnf), the gene with which BLR shares most similarity in Arabidopsis [11], which are not observed in multiple knox 1 mutants [12,13]. Similarly, multiple knox 1 mutants do not resemble blr plants as one role of $B P$ is repression of KNAT2 and KNAT6 [12]. BLR is thus likely to have functions both dependent on and independent of KNOX1.

One $K N O X 1$ independent function of $B L R$ may include control of phyllotaxy as similar defects are not present in knox 1 mutants. Normal phyllotaxy requires correct patterning of cell divisions $[14,15]$ which depend in turn on strictly regulated cell-wall remodelling events [16] themselves driven by mechanical constraints [17]. Primordia outgrowth is accompanied by the de-methyl-esterification of pectic polysaccharides in the cell wall, and perturbation of the methyl-esterification status of pectins within the meristem by altering expression levels of PECTIN METHYLESTERASE 5 has been shown to alter phyllotactic pattern [18]. PME5 has been demonstrated to be negatively regulated by $B L R$ in the meristem dome and this interaction is essential for maintenance of phyllotaxy, as incorrect positioning of organ initiation in blr mutants is suppressed in blr pme5 double mutants. The relationship between $B L R$ and PME5 appears to be context specific as $B L R$ promotes $P M E 5$ expression in internodes [19].

The functions of $B P$ and $B L R$ do overlap in vascular development. Typically in Arabidopsis stems, vascular tissue is radially arranged in collateral bundles with xylem present towards the centre of the stem and phloem positioned towards the outside, such that the xylem, vascular meristem (procambium) and phloem are positioned along the mediolateral axis [20]. Xylem is characterised by the presence of large vessels for water transport and smaller xylary fibres, both of which have secondary cell walls with the adjacent procambium forming an arc of dividing cells. Within the stem, the vascular tissue represents a developmental series with most recently derived tissue at the top and the oldest at the base [20]. A continuous vascular ring has been described in blr lines, a phenotype enhanced in $b p$ blr double mutants [3] by the appearance of undeveloped vascular bundles [3] containing cells previously only observed occurring in small, isolated strips overlying some vascular bundles of $b p$ plants [21,22].

Here we have exploited transgenic plants overexpressing $B L R$ as a tool to identify putative downstream targets of this transcription factor. In addition to the previously described target of BLR, PME5 [19], our microarray analysis has identified misregulation of many other genes encoding cell-wall-associated proteins following $B L R$ over-expression. Defects in cell wall metabolism can affect cell size [23] and we therefore examined blr mutant vascular tissue, where cell size defects have previously been described [3]. We uncovered a genetic interaction between $b l r, b p$ and revoluta (rev), a homeodomain transcription factor of the HD-ZipIII family that has previously been shown to specify adaxial identity, radial patterning in the stem [24] and xylem differentiation [25]. Our results add further support to the notion [19] that in addition to regulating genes at the shoot apex, $B L R$ plays a wider role in development through its control of the extracellular matrix.

\section{Results and discussion}

\section{Over-expression of BELLRINGER reduces organ size}

Over-expression studies have been informative in functional analysis of $K N O X 1$ [26-28]. Using a similar strategy, $B L R$ cDNA was used to generate $35 S:: B L R$ constructs from which $B L R$ over-expression $(B L R-O X)$ transgenic lines were generated. Plants were observed with short hypocotyls, small rosette leaves, narrow cauline leaves, and short siliques (Figure $1 \mathrm{~A}-1 \mathrm{~F}, 1 \mathrm{H}$ ). BLR-OX plants also had shorter, thinner stems than wild type with more axiliary branches (Figure 1G, 1I). Northern blot analysis showed eight lines to exhibit increased $B L R$ levels; $B L R$ transcript levels in two of these lines, $B L R-O X 1$ and $B L R-O X 2$, are shown in Figure 2A.

The organs of BLR-OX lines were smaller than wild type, so differences in cell size and number were investigated in 7 day epidermal hypocotyl cells from $B L R-O X$ and wild type plants (Figure 3A-D). BLR-OX epidermal cells were significantly shorter than controls (Figure $3 \mathrm{~B}$ ); similar reductions were observed in longitudinal sections 

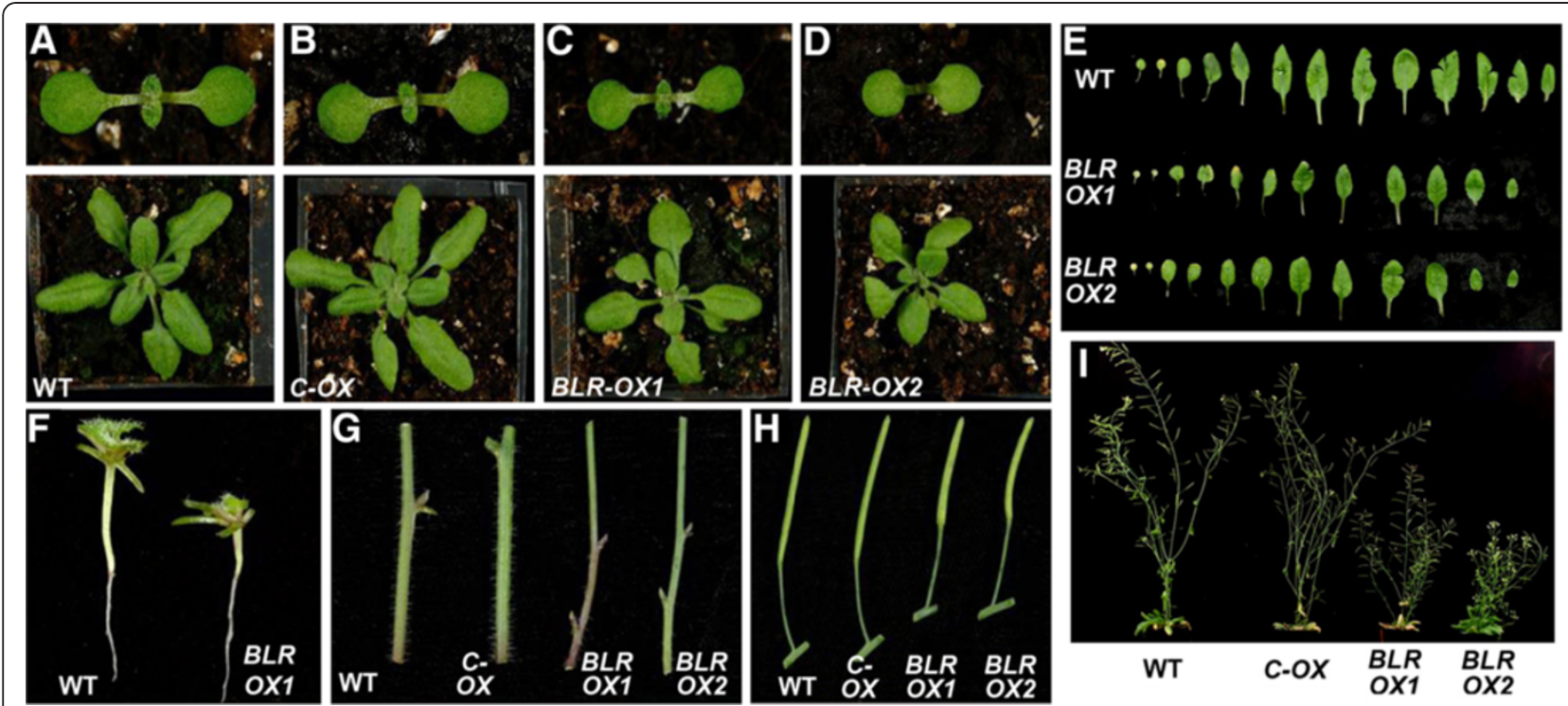

Figure 1 Phenotype of $B L R$-OX lines. 7 day-old (top panel) and 21 day-old (lower panel) wild type (A), transformed control (B), BLR-OX1 (C) and BLR-OX2 (D). (E) Dissected rosette leaves of 4 week-old WT, BLR-OX1 and BLR-OX2 plants. (F) WT and BLR-OX1 hypocotyls at 2 weeks-old. (G-I) Comparison of 5 week-old BLR-OX1,BLR-OX2 and control plant inflorescence stems (G), mature siliques (H) and stature (I).

of inflorescence stem epidermis (Figure 3G-I) and transverse sections of pith parenchyma (Figure 3E-F). This reduction in cell size is thus likely to contribute to the smaller organ size of $B L R-O X$ plants. Other histological features, including those in vascular tissue were indistinguishable from wild type.

\section{Expression changes in BLR-OX plants}

To explore the changes in transcription underlying the $B L R-O X$ phenotype, transcripts from hypocotyls of $B L R-O X$ and control plants were subject to microarray analysis at stage 1.01 [29] of development. Using the 'Significance Analysis of Microarrays' strategy [30], 3109 genes demonstrated consistent expression changes and acceptable spot confidence of which 2957 had appropriate q-values $(<0.1 \%)$. 927 genes demonstrated $>1.5$ fold expression change (Additional file 1: Table S1). Three lines of evidence point to the microarray data reflecting gene expression changes in planta; first, $B L R$ was up-regulated 3.6 fold in $B L R-O X$ compared to Col-0 (Table 1). Second, the expression of $P M E 5$, previously shown to be negatively regulated by $B L R$ is reduced 2.3 fold (Table 1 ) and third, the promoters of 70 of the 100 genes showing the greatest fold change carried predicted BLR consensus DNA binding sites (Table 1). In contrast, the DNA binding sequence for ATHB2, which in common with BLR has a 9 base pair consensus sequence, occurs in only 9 of the 100 promoter regions. The array data was validated by testing expression of MERI-5 (At4g30270; 4.3 fold increase in BLR-OX), a xyloglucan endotransglucosylase which acts to hydrolyze glycosyl bonds, HPRG (At5g65660; 1.6 fold increase), a hydroxyproline-rich plasma membrane localised glycoprotein, and LPT6 (At3g08770; 1.9 fold decrease) and LTPV.2 (At3g53980; 6.3 fold decrease), both of which encode endomembrane localised lipid binding/transport proteins, by northern blot (Figure 2B). In all instances expression reflected the array data.

To understand better the significance of transcriptional differences between $B L R-O X$ and control plants, a gene ontogeny (GO) analysis was carried out. Strikingly, the only significant change proved to be in cell wall associated gene expression, which was enriched from $1.3 \%$ in controls to $6.8 \%$ in BLR-OX lines (Table 1) pointing to a role for BLR in regulating cell wall metabolism or organisation. Changes in cell size are regularly accompanied by expression changes in cell wall associated genes and the $\mathrm{GO}$ analysis thus draws together the gain-of-function phenotypes (Figures 1,3) and the array data (Table 1). Lipid transfer proteins (LTPs) [31] and peroxidases [32] loosen cell walls and induce cell expansion; thus a 6 fold decrease in expression of LTP and a 2.5 fold reduction in peroxidise expression in $B L R-O X$ plants (Table 1 ) is consistent with the reduction in cell size observed in $B L R-O X$ lines. PME5, which is negatively regulated by $B L R$ ( 2.3 fold down-regulated in $B L R-O X)$, alters the gelling properties of cell walls [33], while other genes with altered expression are implicated by homology to have roles in cell expansion. For example members of the thaumatin family (2.8 fold down-regulated) have potential endo- $\beta-1,3$ glucanase activity [34]. 


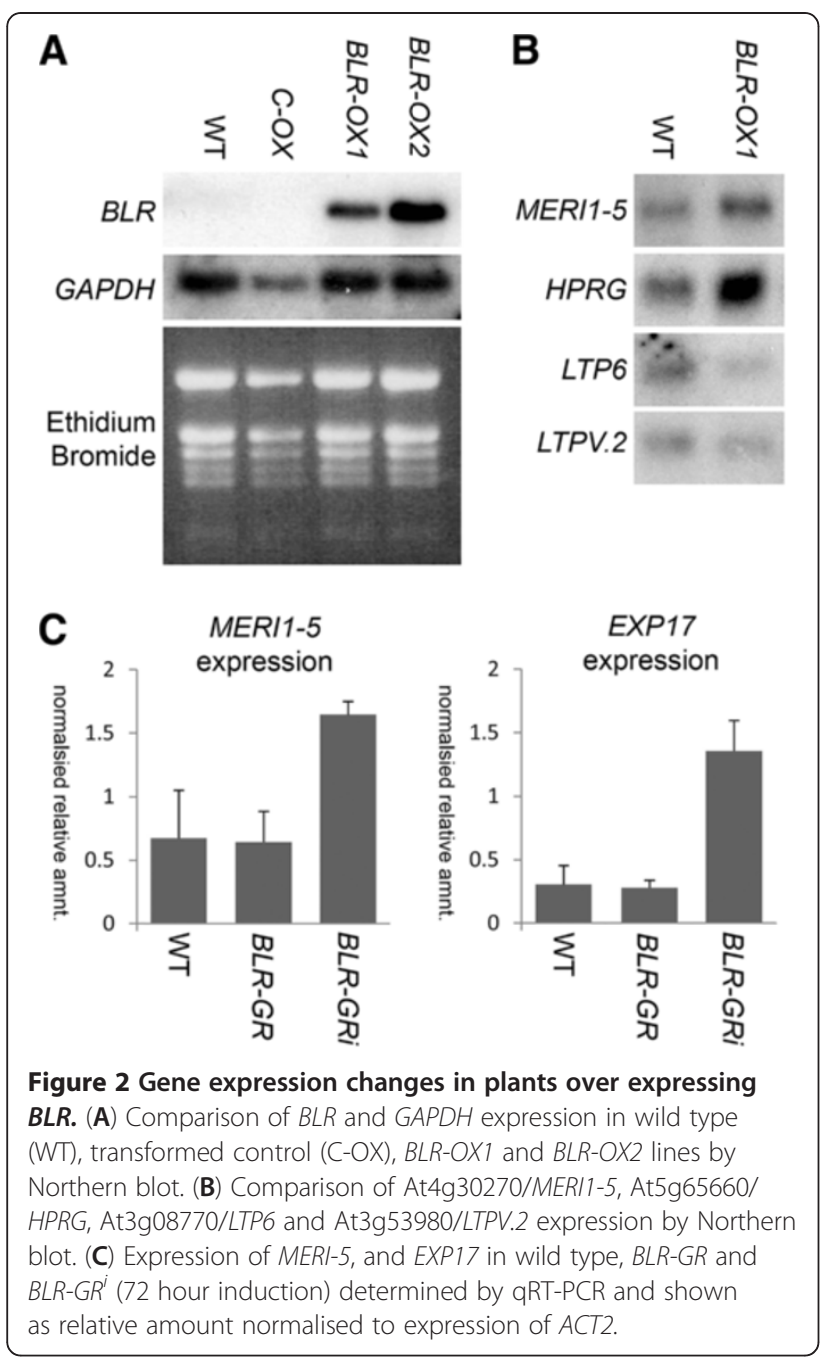

Extreme $B L R$ gain-of-function plants lack organ initiation Organised phyllotaxy, lacking in blr mutants [2-4] requires accurate cell expansion and division during organogenesis [15], processes reliant on close regulation of cell-wall formation and expansion $[16,18]$. We therefore anticipated that phenotypes observed in BLR-OX lines could be strengthened to include disruption of organ initiation due to altered cell wall metabolism, particularly as $B L R-O X$ lines analysed above exhibited only a relatively small expression increase (3.6 fold). Furthermore, in some contexts, BLR is localised in the cytoplasm, and its nuclear localisation is dependent on an interacting partner [2,35]. Plants were therefore generated expressing a steroid-inducible version of $B L R$ in the form of a $B L R-G R$ fusion regulated by the $35 S \mathrm{CaMV}$ promoter which would result in nuclear localisation of BLR in a steroid inducible manner, showing independence of an interacting partner. To confirm that expression changes of cell wall modifying enzymes following $B L R-G R$ induction were consistent with those observed in $B L R-O X$ lines, plants were subjected to dexamethasone (dex) induction for $72 \mathrm{~h}$, as maximum activity of direct transcriptional targets in this system has been demonstrated as occurring between 64 and 128 hours [36]. Following dex induction, expression of both MERI-5 and EXP17 was elevated (Figure 2C), as in BLR-OX lines (Table 1) providing further evidence for $B L R$ regulation of the extracellular matrix.

When $B L R-G R$ seeds were germinated on $10 \mu \mathrm{m}$ dex, only cotyledons were visible after 14 days (Figure 4A), suggesting that high levels of BLR in the nucleus lead to extremely limited growth. This observation may explain the relatively low increases in $B L R$ expression in $B L R$ $O X$ as plants with higher expression levels may not have been recovered in the T1 generation. Seedlings germinated for 3 days in the absence of steroid and subsequently transferred to $10 \mu \mathrm{m} \operatorname{dex}\left(B L R-G R^{i}\right)$, produced leaves with severely reduced expansion (Figure 4B-E). Visible leaves no longer emerged after 21 days and the apex became enlarged (Figure 4F), suggesting that growth continued in the absence of organ initiation. Sections cut through these enlarging $B L R-G R^{i}$ apices revealed an absence of lateral organ primordia or any other clearly differentiated cell types (Figure 4G-H), suggesting that high levels of nuclear BLR results in anisotropic growth.

Normal organ initiation and phyllotaxy in Arabidopsis requires interplay between $B L R$ repression of $P M E 5$ in the shoot apical meristem [19], and loss of this repression during formation of organ primordia [4]. Loss of PME5 repression has been proposed to allow other cell wall modifying components to access the wall. Our results support this hypothesis for, in our $B L R-G R^{i}$ plants, $B L R$ would continue to negatively regulate $P M E 5$ with the consequent inhibition of organ initiation we observed.

Organ initiation requires both the correct patterning of cell divisions $[14,15]$ and strictly regulated cell wall remodelling $[16,18]$. The organisation of cell divisions in the apex has been shown to depend on mechanical pressures [17], which are likely to be influenced by the cell wall [37]. The current model for cell-wall remodelling during organ initiation at the apex holds that the breakage and annealing of cell-wall components is controlled by connections with microtubules, possibly through regulation of cellulosemicrofibril length [38]. Treatment of Arabidopsis apices with oryzalin, which depolymerises microtubules and prevents ordered division, results in a bulbous apex and loss of lateral-organ initiation when combined with disrupted auxin signalling [39]. The cell wall plays a central part in the transmission of chemical and mechanical signals between cells, and the positioning of developmental cues at the apex of sunflowers is reported to be sensitive to mechanical interventions [17]. Our data suggest that BLR modulates the expression of genes encoding cell wall associated proteins, and our observation that plants strongly over- 

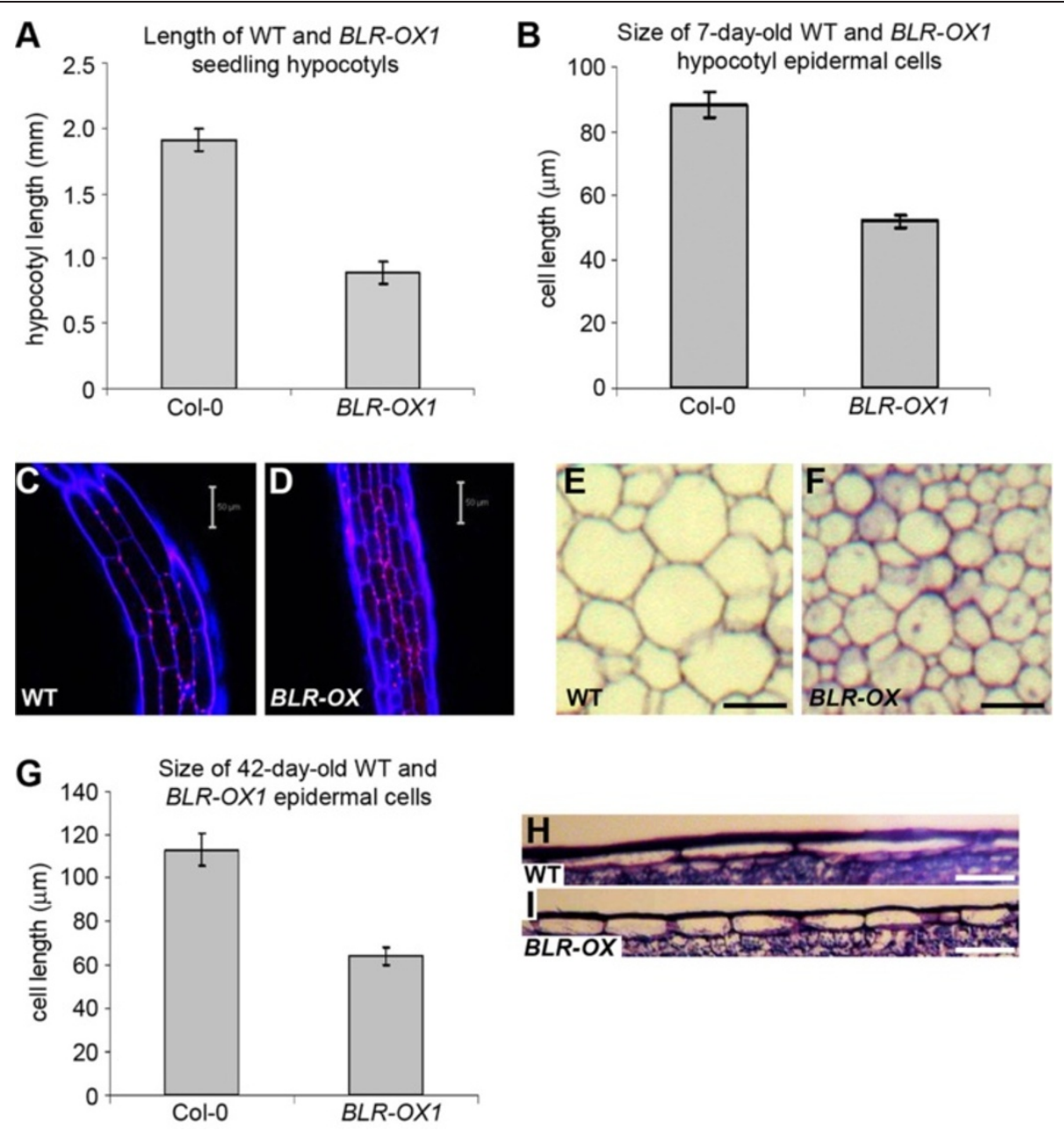

Figure 3 Cell size defects of BLR-OX seedlings. (A) Average hypocotyl length of 7 day-old wild type and BLR-OX1 lines ( $n=14)$. (B) Mean epidermal cell size in hypocotyls of 7 day-old plants $(n=33) .7$ day-old wild type $(\mathbf{C})$ and BLR-OX (D) hypocotyl epidermis stained with propidium iodide. $200 \mu \mathrm{M}^{2}$ cross-section from 42 day-old wild type (E) and BLR-OX1 (F) stems showing parenchyma cells. (G) Mean epidermal cell size of 42 day-old plants $(n=41) .100 \mu \mathrm{M}$ row of epidermal cells from 42 day-old wild type $(\mathbf{H})$ and BLR-OX (I) stems. Error bars show standard error. Scales are $50 \mu \mathrm{m}(\mathrm{E}-\mathrm{F})$, or $10 \mu \mathrm{m}(\mathrm{H}-\mathrm{I})$.

expressing $B L R-G R^{i}$ fusions generate bulbous apices incapable of forming lateral organs is consistent with the view that closely-regulated cell wall development is essential for ordered initiation of lateral organs at the shoot apex.

\section{rev enhances the bp blr phenotype}

The array experiments identified genes involved in cell expansion to be misregulated in BLR-OX plants. Expression changes in genes involved in cell expansion are also a feature of ectopic knoxl (STM) expression in poplar stem vascular tissue [40], and several putative $B L R$ targets identified in $B L R-O X$ arrays are reciprocally regulated in $b p$ mutants [41] (Table 1). BP is required for patterning the inflorescence stem as sectors of differentiation-defective epidermal, cortical and vascular cells are present in $b p$ mutant stems $[22,42,43]$. Furthermore, $B P$ negatively regulates biosynthesis of lignin [41], a cell wall polymer, that is present in cells that have secondary walls including those of the vascular tissue. Despite exhaustive analysis, blr and wild type hypocotyls proved indistinguishable (not shown); however we saw clear differences in the vascular tissue of the inflorescence stem where BLR is expressed [4]. Loss of function blr phenotypes have been described in this region [3] in the relatively newly formed vascular tissue at the top of the stem (between the third and fourth siliques). Here blr mutants were found to form a continuous ring of vascular tissue, while $b p$ blr double mutants demonstrated an enhanced blr phenotype as cells in the continuous vascular ring failed to differentiate and contained small, densely stained cells where xylem cells with secondary cell walls formed in wild type plants [3].

We analysed vascular tissue at a later stage in development, adjacent to the first cauline leaf on the primary inflorescence stem. blr vascular bundles were patterned 
Table 1 Genes prominently mis-regulated in BLR-OX

\begin{tabular}{|c|c|c|c|c|}
\hline \multirow[t]{2}{*}{ AGI number } & \multirow[t]{2}{*}{ Common name } & \multicolumn{3}{|c|}{ Expression } \\
\hline & & $B L R-O X$ & WT & Fold Change \\
\hline \multicolumn{5}{|c|}{ UPREGULATED IN BLR-OX } \\
\hline At1g10070 1,4 & Branched-chain amino acid aminotransferase 2 (BCAT-2) & $469 \pm 320$ & $47 \pm 12$ & 10.0 \\
\hline At4g30270 1,2,4 & Xyloglucan endotransglucosylase/hydrolase 24 (MERI-5) & $2269 \pm 601$ & $522 \pm 38$ & 4.3 \\
\hline At3g47340 1,2,4 & Glutamine dependent aparagine synthetase 1 & $1,231 \pm 470$ & $281 \pm 41$ & 4.4 \\
\hline At1g75750 1,2,4 & GA-responsive GAST1 protein homolog (GASA1) & $10890 \pm 4769$ & $2641 \pm 390$ & 4.1 \\
\hline At5g02030 1,4 & BELLRINGER & $2507 \pm 452$ & $706 \pm 1,23$ & 3.6 \\
\hline At2g19800 1,4 & Myo-inositol oxygenase 2 (MIOX2) & $2104 \pm 759$ & $700 \pm 357$ & 3.0 \\
\hline At5g56870 1,4 & Beta-galactosidase 4 (BGAL4) & $842 \pm 140$ & $265 \pm 57$ & 3.2 \\
\hline At2g30600 2,4 & BTB/POZ domain protein involved in cell adhesion & $2624 \pm 839$ & $1110 \pm 353$ & 2.4 \\
\hline At5g49360 ${ }^{1,4}$ & $\beta$-D-xylosidase/a-L-arabinofuranosidase (BXL1) & $2105 \pm 591$ & $983 \pm 484$ & 2.1 \\
\hline At4g35770 ${ }^{2}$ & Senescence-associated/Dark inducible protein (SEN1/DIN1) & $2393 \pm 413$ & $1,206 \pm 374$ & 2.0 \\
\hline At2g45170 2,4 & Autophagy 8e (APG8e) & $3685 \pm 133$ & $2007 \pm 193$ & 1.8 \\
\hline At4g36850 2,4 & PQ-loop repeat family/transmembrane family protein & $503 \pm 88$ & $270 \pm 45$ & 1.9 \\
\hline At5g07440 2,4 & Glutamate dehydrogenase 2 (GDH2) & $4403 \pm 685$ & $2364 \pm 237$ & 1.9 \\
\hline At4g01630 1,4 & Expansin, putative (EXP17) & $162 \pm 24$ & $88 \pm 17$ & 1.8 \\
\hline At5g11720 ${ }^{1}$ & Glycoside hydrolase family 31 & $1054 \pm 113$ & $578 \pm 85$ & 1.8 \\
\hline At1g53910 1,4 & AP2 domain-containing protein RAP2.12 & $9167 \pm 47$ & $5043 \pm 441$ & 1.8 \\
\hline At1g12780 1,2 & UDP-D-glucose/UDP-D-galactose 4-epimerase 1 (UGE1) & $239 \pm 43$ & $136 \pm 35$ & 1.8 \\
\hline At4g14130 ${ }^{1}$ & xyloglucan endotransglycosylase-related protein (XTR7) & $41,2 \pm 156$ & $272 \pm 105$ & 1.5 \\
\hline At1g35250 1,4 & Thioesterase superfamily protein & $1164 \pm 132$ & $769 \pm 91$ & 1.5 \\
\hline \multicolumn{5}{|c|}{ DOWNREGULATED IN BLR-OX } \\
\hline At3g53980 1,4 & Protease inhibitor/seed storage/lipid transfer protein family (LTPV.2) & $282 \pm 35$ & $1776 \pm 616$ & 6.3 \\
\hline $\operatorname{At1g73620~}^{1}$ & Thaumatin-like protein, putative & $268 \pm 23$ & $741 \pm 23$ & 2.8 \\
\hline At1g21890 1,4 & Nodulin MtN21/EamA-like transporter family protein & $165 \pm 51$ & $403 \pm 81$ & 2.4 \\
\hline At5g06390 ${ }^{1,4}$ & FASCICLIN-like arabinogalactan protein 17 (FLA17) & $65 \pm 11$ & $161 \pm 6$ & 2.5 \\
\hline At5g67400 1,4 & Root hair specific 19/Peroxidase 73 (RHS19/PER73) & $153 \pm 8$ & $377 \pm 37$ & 2.5 \\
\hline At5g47500 1,4 & Pectin lyase-like superfamily protein (PME5) & $302 \pm 93$ & $687 \pm 136$ & 2.3 \\
\hline At3g05600 1,4 & 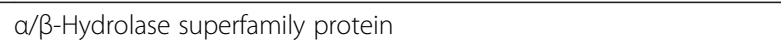 & $58 \pm 1,2$ & $116 \pm 13$ & 2.0 \\
\hline At1g62560 1,3 & Flavin-monooxygenase glucosinylate S-oxygenase 3 (FMO GS-OX3) & $406 \pm 102$ & $775 \pm 194$ & 1.9 \\
\hline At3g08770 ${ }^{1,4}$ & Lipid transfer protein 6 (LTP6) & $1475 \pm 1,25$ & $2758 \pm 98$ & 1.9 \\
\hline At3g05470 1,4 & Actin-binding FH2 (formin homology 2) family protein & $275 \pm 160$ & $516 \pm 311$ & 1.9 \\
\hline At5g01870 ${ }^{1}$ & Lipid transfer protein 10 (LTP10) & $360 \pm 24$ & $638 \pm 58$ & 1.8 \\
\hline At5g61020 1,4 & evolutionarily conserved C-terminal region 3 (ECT3) & $421 \pm 98$ & $736 \pm 160$ & 1.7 \\
\hline At1g20930 1,4 & Cyclin-dependent kinase B2;2 (CDKB2;2) & $359 \pm 61$ & $598 \pm 66$ & 1.7 \\
\hline
\end{tabular}

Genes with putative roles in extracellular matrix regulation (GO analysis), altered expression in bp mutants [36] or having a BLR consensus binding site in the promoter (http://arabidopsis.med.ohio-state.edu/AtcisDB/) are shown.

${ }^{1}$ Role, or putative role in modification of the extracellular matrix.

${ }^{2}$ Reduced expression in bp compared to wild-type plants (Mele et al., 2003).

${ }^{3}$ Increased expression in $b p$ compared to wild-type plants (Mele et al., 2003).

${ }^{4}$ BLR DNA binding consensus sequence present in promoter.

Expression values are presented as the mean \pm standard error for three biological replicates.

differently from those of wild type, as the arc of procambial cells present in wild type appeared shallower in blr (Figure 5A, C). blr mutants with the strongest phenotypes also had vascular bundles lacking large xylem vessels and containing apparently only xylary fibres, suggesting that there was either a failure of vessel specification, or a defect in vessel cell size (Figure 6A, C). 'Stripes' of differentiation- defective cells overlying some vascular bundles in $b p$ mutants gave rise to two distinct vascular morphologies (Figures 5B, 6B); Within the stripes xylem differentiation was altered because as with blr mutants, xylem lacked large vessels, however, in contrast to blr mutants, many of the smaller cells had not generated secondary cell walls $[22,42]$ (Figure 6B). In areas outside the differentiation defective 

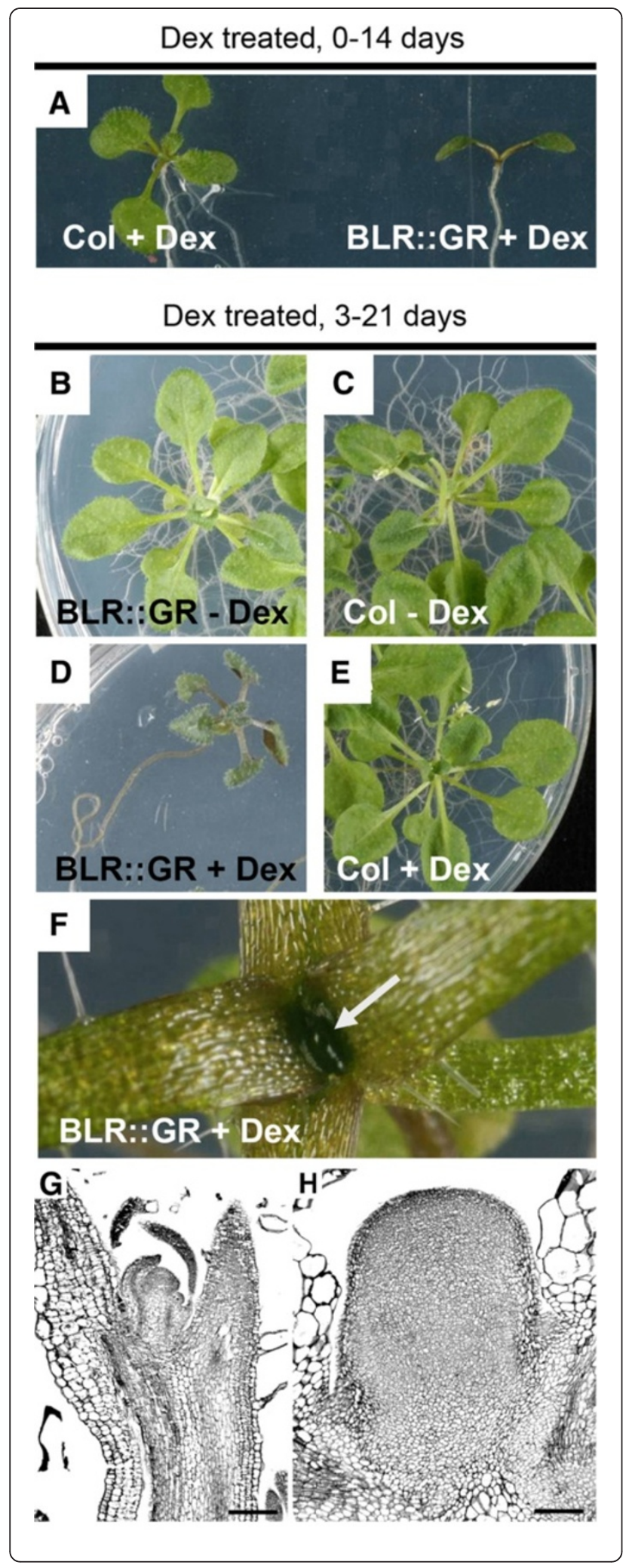

Figure 4 Phenotypes of 35S::BLR-GR Plants. (A) Plants expressing $B L R-G R$ did not generate post-embryonic structures when germinated on plates containing Dex. (D-H) Plants treated with dex, 3 days post-germination. $B L R-G R^{i}(\mathbf{D}, \mathbf{F})$ did not initiate new lateral organs but developed a bulbous structure (arrow in $\mathbf{F}$ ) at the apex, whereas dex treated wild type $(\mathbf{E})$ and untreated $B L R-G R(\mathbf{B})$ or wild type (C) developed normally. Longitudinal sections through apices $(\mathbf{G}-\mathbf{H})$ shows that in wild type plants at 3 weeks-old, the programme of inflorescence development had initiated $(\mathbf{G})$, but induced 35S::BLR:::GR plants lateral organ initiation had ceased $(\mathbf{H})$, Scales are $100 \mu \mathrm{m}$.

stripe, vascular patterning was indistinguishable from wild type (Figure $5 \mathrm{~B}$ ).

$b p b l r$ vascular tissue has been described as reminiscent of that present in differentiation defective areas of $b p$ mutants [3]. In $b p$ blr bundles outside the differentiationdefective stripe, small darkly staining cells as described by Smith et al. [3], were observed towards the centre of the stem (Figure 5F) suggesting that these cells were derived early in vascular development. However, xylem cells generated later in development (closest to the procambium) underwent significant differentiation in $b p$ blr double mutants because secondary cell walls were present, but as with extreme blr mutants, all xylem cells were reminiscent of xylary fibres (Figure 6D). In contrast, we observed very few xylem cells with secondary walls in $b p$ blr vascular bundles within the differentiation-defective tissue associated with the $b p$ mutation, even at this late stage of development (Figures 5F, 6D). Consequently, and consistent with other reports [3], blr enhances $b p$ in the context of the vascular tissue and this interaction is particularly evident in mature $b p$ blr vascular tissue within differentiation-defective regions of the stem (Figures 5A-C, 5F, 6A-D). We reasoned that analysis of $b p$ and $b l r$ mutant combinations in a background of altered xylem identity may reveal redundant functions of $b p$ and $b l r . R E V$ has been associated with radial patterning in the stem [24] and has previously been shown to be required for development of interfascicular fibres $[25,44]$, for xylem differentiation and maintenance of xylem cell number [25]. The role of $R E V$ in the xylem is likely to be partially redundant as phenotypes appear enhanced in rev phb/+ or rev phv mutants [45]. Furthermore in the shoot apex, as with $b p$ [13] and blr [2], rev enhances the phenotype of weak alleles of stm [46] suggesting that $R E V$ may have overlapping functions with $B L R$. We therefore examined inflorescence stem vascular tissue in $b p, b l r$ and rev combinatorial mutants. Within rev vascular bundles xylem tissue resembled wild type in that both vessels and parenchyma were present, however the arc of procambium was shallower as in blr plants (Figure 5D), a phenotype also occurring in bp rev mutant vascular tissue, outside of differentiation defective tissue (Figure 5G). Within differentiation defective tissue, the xylem cells were generally similar to those of $b p$ single mutants except that the relatively few 


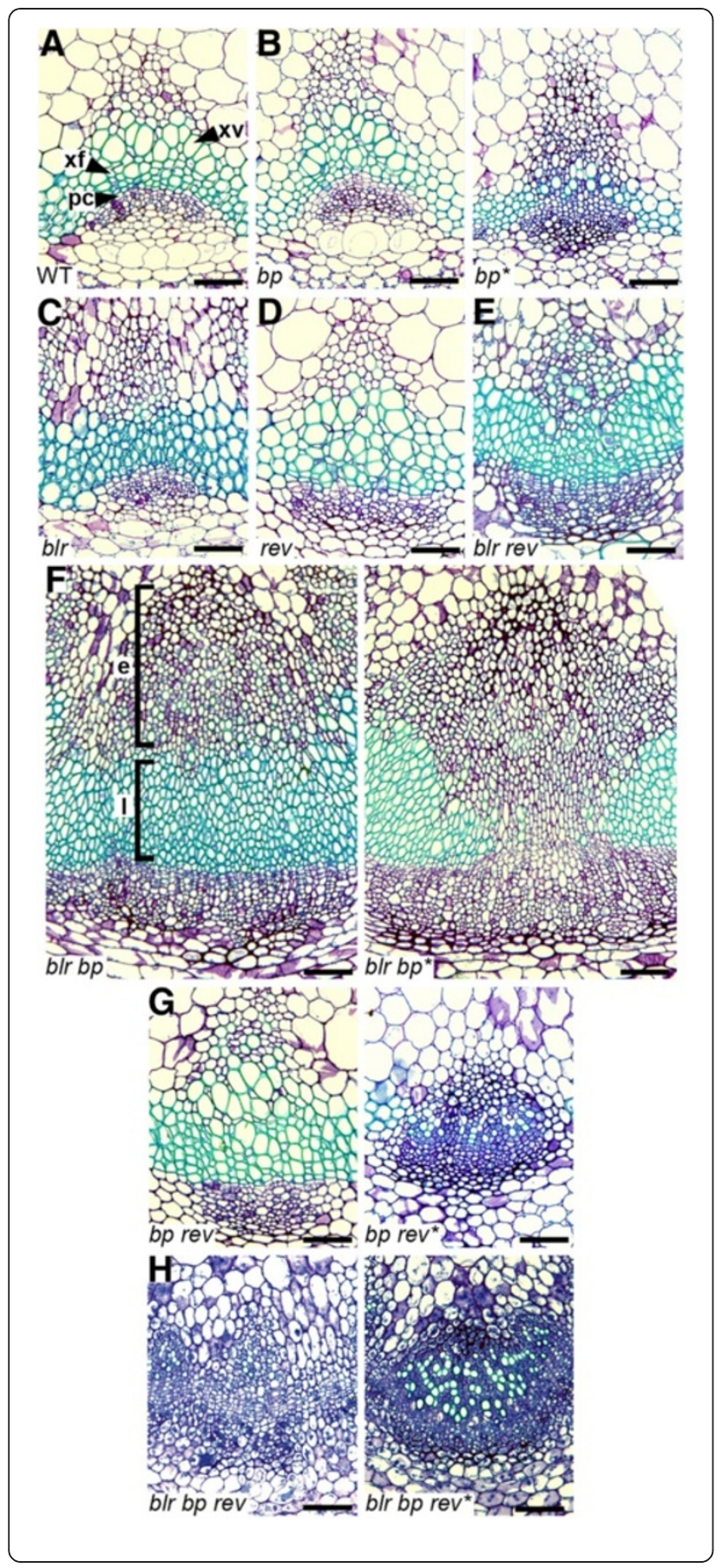

cells that developed secondary walls were extremely small compared with those of $b p$ (Figure 6F). blr rev and blr vascular bundles were similar but, strikingly, the xylem in vascular bundles of blr bp rev triple mutants comprised small and darkly stained xylem cells, whether or not the bundles lay within the stripe of differentiation-defective cells (Figures 5H, 6G). In extreme cases, no cells with secondary walls were seen in vascular bundles (Figure $5 \mathrm{H}$ ).

Genetic interactions between $B L R, R E V$ and $B P$ in the stem vary depending on tissue type. Vascular cell division

\section{Figure 5 Vascular tissue in $b l r, b p$ and rev mutant}

combinations. Toluidine blue stained sections from inflorescence stem vascular tissue adjacent to the first cauline leaf. (A) Wild type vascular bundle with arrowheads pointing to a xylem vessel (xv), xylary fibres (xf) and vascular meristem (procambium; pc). (B) bp mutants are indistinguishable from wild type, except in differentiation defective stripes $\left(b p^{*}\right)$ where the arc of procambium cells is flattened, xylem vessels are absent or reduced in size and many cells are small and darkly stained suggesting they lack secondary walls (green in wild type xylem; A). blr (C) and blr rev (E) vascular tissue in which the arc of procambium is flattened and xylem vessels appear absent. (D) rev. (F) Large vascular expansion in blr bp. In vascular tissue derived early in development (square bracket; e) all cells are small and many lack secondary walls. In contrast, later derived tissue (square bracket; I) contains

differentiated xyary fibres but no vessels, except in differentiation defective regions of the stem (blr $\left.b p^{*}\right)$ where very few cells with secondary wall are present. (G) bp rev double mutants are indistinguishable from rev in most regions of the stem, however, in differentiation defective regions ( $b p r^{*} v^{*}$ ) cells were smaller than those observed in $b p^{*}$. (H) Cells in blr bp rev inflorescence stems were extremely small and in the vast majority of cases secondary walls appeared absent. Scales are $50 \mu \mathrm{m}$.

between vascular bundles is one of the hallmarks of secondary growth (radial expansion). $B P$ and $B L R$ repress this process as $b p$ blr mutants have a continuous ring of vascular tissue [3]. However, $R E V$ appears to be required for the large increase in vascular tissue present in $b p$ blr because $b p$ blr rev lines demonstrate a reduction in vascular tissue compared to $b p$ blr (Figure 5F, H). This observation supports a role for $R E V$ (in contrast to $B P$ and $B L R$ ) in promoting secondary growth, a function that has previously been suggested for its poplar orthologue [47]. BLR, REV and $B P$ do not have contrasting roles in regulating xylem differentiation. The presence of xylem cells with secondary walls was reduced in double and triple mutant combinations (Figure 6) suggesting that $B L R, R E V$ and $B P$ act together in this process. Consequently, $B L R, R E V$ and $B P$ interactions are context dependent.

Although the primary defect observed in $B L R-O X$ was one of reduction in cell size, similar to that observed in vascular tissue of blr mutant combinations, the relationship between cell size and expression of particular cell wall modifying genes is not always straightforward [48-53]. For example, over-expression of an E2Ff transcription factor causes a reduction in cell size, accompanied by a decrease in expression of expansins (EXP3, 7 and 9), a UDP-glucose-glucosyl transferase (UGT) and $M E R I-5$ [52] (up-regulated 4.6 fold in BLR-OX). However, over-expression of $M E R I-5$ is reported elsewhere as promoting precocious elongation [54]. BLR control of the extracellular matrix is thus likely to be similarly complex. BLR-OX plants have smaller cells than those of wild-type, but blr mutants, when combined with $b p$ and rev also resulted in vascular tissue with smaller cells. 


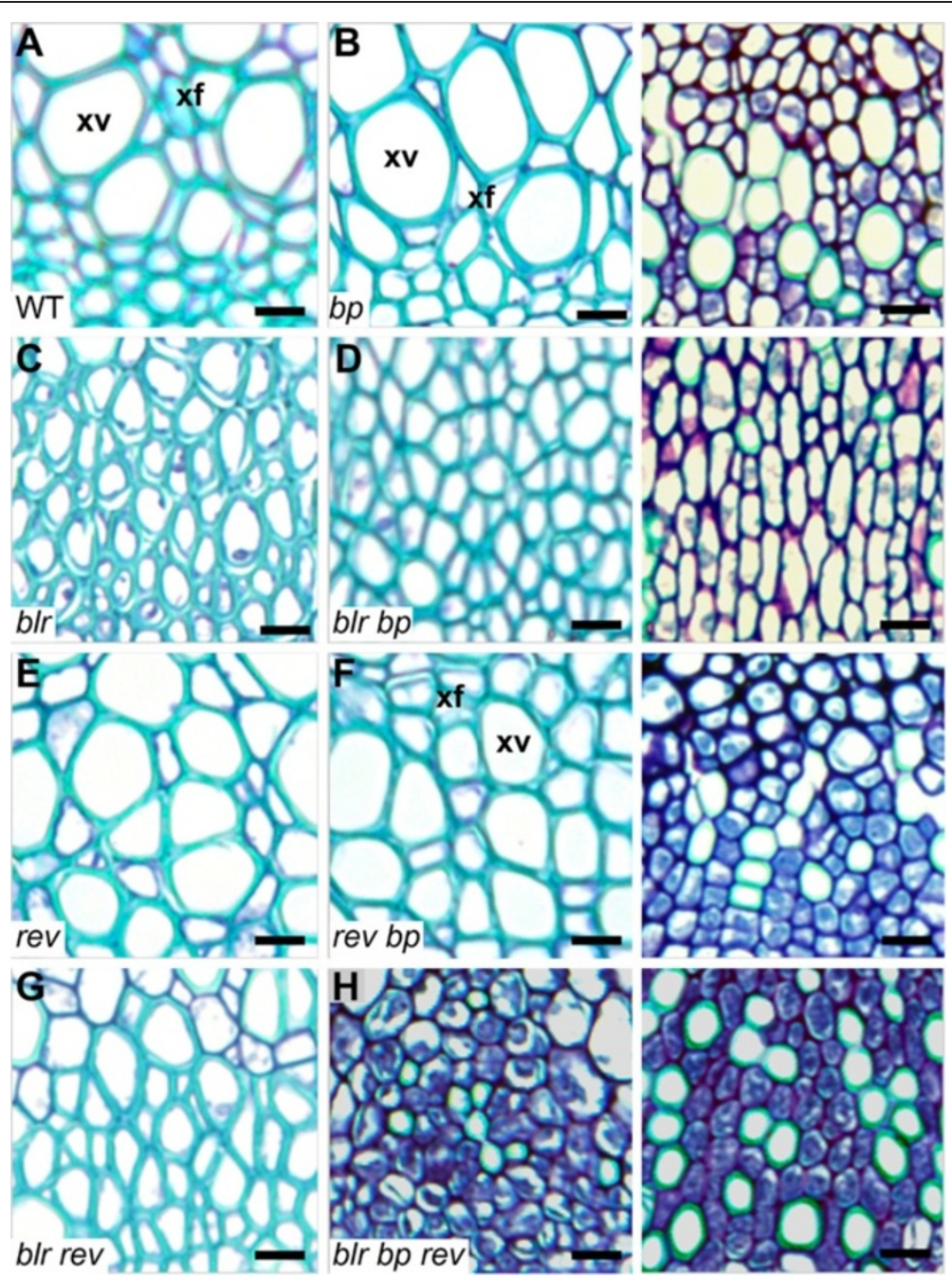

Figure $6 \mathbf{b l r}, \mathbf{b p}$ and $\mathbf{r e v}$ mutant xylem. Xylem from vascular bundle in inflorescence stems. (A) Wild type xylem with vessels (xv) and xylary fibres ( $\mathrm{xf}$ ) marked. (B) bp mutant xylem is indistinguishable from wild type (left hand side; LHS), except in differentiation defective tissue (right hand side; RHS) where vessels are absent or reduced in size and many cells lack secondary walls (green in wild type xylem; A). (C) Extreme blr phenotype where xylem vessels appear absent. (D) blr bp xylem in later derived tissue (see Figure 5F) with xyary fibres but no vessels (LHS). In differentiation defective regions of the stem (RHS) Few cells with secondary wall are present. (E) rev xylem, similar to wild type. (F) bp rev xylem is like that of rev (LHS) except in differentiation defective regions (RHS) where cells were smaller than those observed in bp. (G) rev blr xylem. (H) Most blr bp rev xylem lacked secondary walls. Scales are $10 \mu \mathrm{m}$.

\section{Conclusions}

Control of cell expansion is essential for normal plant development. Our results demonstrate that $B L R$ controls cell expansion and differentiation in vascular development in a process also involving $B P$ and $R E V$ transcription factors. Microarray analysis of plants over-expressing $B L R$ indicates that $B L R$ targets many genes involved in cell wall regulation. As dynamic control of the cell wall is essential for cell expansion, we suggest that the striking phenotypes of plants over- and under-expressing BLR may result from the disruption of this process.

Closely regulated cell wall dynamics are also required for a normal pattern of organ initiation at the shoot apex and this process has previously been shown to be regulated by
$B L R$ repression of PME5 [19]. Our experiments with BLR$G R$ suggest that further transcriptional targets of $B L R$ may also play important parts in this process.

\section{Methods}

\section{$B L R$ overexpression}

Full-length $B L R$ cDNA was cloned into pGEM-T Easy (Promega), subcloned as an EcoRI fragment into pART7 [55] and sequenced to identify plasmids with $B L R$ in the sense orientation. The resultant CaMV35S::BLR::OCS-terminator cassette was subsequently excised from pART7 using Not I and subcloned into pMLBART. The resultant $B L R-O X$ binary vector was introduced into Arabidopsis (Col-0) using standard methods [56]. For steroid- 
inducible $B L R$ lines, two PCR products were amplified, one containing $B L R \mathrm{cDNA}$ and a second containing the glutocoticoid receptor (GR). The GR forward primer (AAAAAGCAGGCTTAATGACCACACTCAACATG) contained a partial attB1 site, while the reverse primer (GCTCCACCTCCACCTCCATACTCATGG) contained an Ala-Gly linker and part of the $5^{\prime} B L R$ sequence. Similarly, the forward $B L R$ primer (GGAGGTGGAGGTG GAGCATGGCTGATGCAT) contained the Ala-Gly linker while the reverse primer (AGAAAGCTGGGTTCAACC TACAAAATCATG) contained an attB2 site. The two PCR products were mixed in equimolar concentrations and used as templates for overlap PCR with attB1 and attB2 primers resulting in a $B L R-G R$ in-frame fusion, which was cloned into pDONR Zeo, sequenced, and subcloned into pEarleyGate100 using Gateway technology. BASTA-resistant Col-0 transgenic lines were generated as described above, and Arabidopsis transformants that had a wild-type phenotype when uninduced but responded to $10-\mu \mathrm{M}$ dexamethasone were used for subsequent analysis.

\section{Gene expression analysis}

For Microarray experiments total RNA was extracted from Col-0 and BLR-OX plants using an RNeasy Plant Mini Kit (Qiagen). RNA integrity was checked on a 2100 BioAnalyzer (Agilent, CA, USA) prior to cDNA synthesis from $2 \mu \mathrm{g}$ total RNA using a 3DNA Array $900^{\mathrm{TM}}$ kit (Genisphere Inc., Hatfield, PA, USA) with RT-primers that contained a dendrimer [57] capture sequence. Labelled cDNAs were hybridised for 16 hours to the Arabidopsis Atv3.3.2.z Array printed with the Operon Arabidopsis thaliana version 3 Array-Ready Oligo Set (Galbraith, USA) using a Slidebooster (Advalytix, Brunnthal, Germany) with a power setting of 27 and a pulse setting of 7:3. Array slides were washed in $2 \times \mathrm{SSC}, 0.2 \% \mathrm{SDS}\left(10 \mathrm{~min}, 55^{\circ} \mathrm{C}\right), 2 \times \mathrm{SSC}(10 \mathrm{~min}$, Room temp.) and $0.2 \times$ SSC (10 min, room temp.). Fluorescent-labelled Genisphere 3DNA dendrimers containing approximately $850 \mathrm{Cy} 3$ or $\mathrm{Cy} 5$ dyes were hybridized for 4 hours to the arrays. Following a set of washes as above, arrays were scanned using a ScanArray Express HT Microarray Scanner (Perkin Elmer).

After calibration to obtain scans within the optimum dynamic range, image analysis and spot feature quantification were performed using BlueFuse version 3.1 (BlueGnome, Cambridge, UK). Data from each slide were confirmed to be essentially linear and globally median-normalized before further analysis. Spots were excluded when the combined confidence values of biological replicates was $<0.005$. Microarray data were median-normalized in $\mathrm{Cy} 5$ and $\mathrm{Cy} 3$ channels. Spots that showed expression changes in the same direction in all three replicates were analysed further.

Normalized Cy5/Cy3 $\log _{2}$ ratios (three values for each gene for BLR-OX lines and three for Col-0) were used to perform a significance analysis of microarray (SAM) [30] using the TIGR Multi Experiment Viewer (TMEV V3.0.3). The imputation engine was set as 10-nearest neighbour imputer and the number of permutations was 100 . The delta value in the Significance Analysis was adjusted so that estimated false discovery rate (FDR) was $0 \%$ for significant genes. The expression-fold change was set as 1.5 as a threshold for changes of potential biological importance. The microarray data described here have been submitted to MIAMExpress (http://www.ebi.ac.uk/arrayexpress/ experiments/E-MEXP-852). GO annotation analysis was performed using http://www.arabidopsis.org/tools/bulk/go/ index.jsp.

For northern blot analysis of primary transformants, two micrograms of total RNA extracted from leaf tissue was subjected to electrophoresis on a $1.2 \%$ formaldehyde agarose gel, which was subsequently blotted onto Hybond-NX membrane and hybridised with a full-length ${ }^{32} \mathrm{PdCTP}-$ labelled cDNA probe. RNA was also visualised by ethidium bromide staining and the filter hybridised with GAPDH as loading controls. Validation of microarray targets was carried out on RNA from 6-7 day-old seedlings. Primers were used to amplify the $3^{\prime}$ UTR and adjoining exons for specific probes, which were ${ }^{32} \mathrm{PdCTP}$-labelled.

qRT-PCR analysis was carried out using SYBR Green JumpStart Taq ReadyMix (Sigma) and an ABI Prism 7000 machine (Applied Biosystems) with the standard sybr green detection programme. A melting curve was produced at the end of every experiment to ensure that only single products were formed. Gene expression was determined using a version of the comparative threshold cycle $(\mathrm{Ct})$ method. The average amplification efficiency of each target was determined using LinReg [58] and samples were normalised to $18 S$ rRNA (not shown) and ACT2 (shown). Results were similar independent of control used. Samples were measured in technical triplicates on biological triplicates.

\section{Plant materials, growth conditions and imaging}

Plants were grown in long-day conditions at $20^{\circ} \mathrm{C}$. Mutant lines, blr (van-1) [2], rev-6 [46], and bp-1 [22,42], have been previously described. Digital images of the plants were analysed in Scion Image (Scion Corporation, Maryland, USA) for hypocotyl measurements. For histological analysis of $B L R-O X$ and $B L R-G R$, plants were fixed, wax-embedded and sectioned using standard methods. Sections between 6 and 10 micrometers were stained with toluidine blue for light microscopy. For visualisation of hypocotyl epidermal cells, seedlings were stained with $10 \mu \mathrm{g} \mathrm{ml}^{-1}$ propidium iodide (Molecular Probes Europe BV, Netherlands) for 10 minutes in the dark before transfer to water. Seedlings were viewed by confocal microscopy on a Zeiss (Welwyn, UK) CLSM 510.

Toluidine blue stained resin sections for visualisation of transverse vascular bundle sections in inflorescence 
stems were taken from 5 week old plants. Sections were taken above the first cauline leaf and prepared as described in [59].

\section{Additional file}

Additional file 1: Genes changed in expression in BLR-OX in the same direction in all 3 replicates with an average of $>1.5$-fold change, arranged in order of spot confidence.

\section{Authors' contributions}

$A M B$ and HGD designed the experiments. JPE, LM, WZJ, HP, NC, NS and AMB carried out the experiments. JPE, AMB and HGD wrote the manuscript. All authors read and approved the final manuscript.

\section{Authors' information}

Anuj M Bhatt and Hugh G Dickinson Joint last authors.

\section{Acknowledgements}

We thank Andrey Lagodienko for preliminary BLR-OX analysis, lan Moore for confocal microscopy assistance, John Baker for photography and Jo Dowding for technical help. Seeds were from NASC and materials for microarrays were provided by the University of Arizona. This study was supported by BBSRC (grant BB/G18339). Microarray facilities were established with financial support from the EPA Cephalosporin Trust (NS)

\section{Author details}

'Department of Plant Sciences, University of Oxford, South Parks Road, Oxford, UK. ²Department of Pathology, University of Oxford, South Parks Road, Oxford, UK. ${ }^{3}$ Faculty of Life Sciences, University of Manchester, Manchester, UK. ${ }^{4}$ Present address: Department of Biochemistry, University of Nebraska, Lincoln, NE, USA. ${ }^{5}$ Present address: Agilent Technologies, Wokingham, UK. ${ }^{6}$ Present address: Centre for Systems and Synthetic Biology, Brunel University, London, UK.

Received: 19 January 2012 Accepted: 17 September 2012 Published: 13 November 2012

\section{References}

1. Roeder AHK, Ferrandiz C, Yanofsky MF: The Role of the REPLUMLESS Homeodomain: Protein in Patterning the Arabidopsis Fruit. Curr Biol 2003, 13(18):1630-1635.

2. Bhatt AM, Etchells JP, Canales C, Lagodienko A, Dickinson H: VAAMANA-a BEL1-like homeodomain protein, interacts with KNOX proteins BP and STM and regulates inflorescence stem growth in Arabidopsis. Gene 2004, 328:103-111.

3. Smith HMS, Hake S: The Interaction of Two Homeobox Genes, BREVIPEDICELLUS and PENNYWISE, Regulates Internode Patterning in the Arabidopsis Inflorescence. Plant Cell 2003, 15(8):1717-1727.

4. Byrne ME, Groover AT, Fontana JR, Martienssen RA: Phyllotactic pattern and stem cell fate are determined by the Arabidopsis homeobox gene BELLRINGER. Development 2003, 130(17):3941-3950.

5. Bao X, Franks RG, Levin JZ, Liu Z: Repression of AGAMOUS by BELLRINGER in Floral and Inflorescence Meristems. Plant Cell 2004, 16(6):1478-1489.

6. Smith HMS, Boschke I, Hake S: Selective interaction of plant homeodomain proteins mediates high DNA-binding affinity. Proc Natl Acad Sci USA 2002, 99(14):9579-9584.

7. Vollbrecht E, Veit B, Sinha N, Hake S: The developmental gene Knotted-1 is a member of a maize homeobox gene family. Nature 1991, 350(6315):241-243.

8. Lee J-H, Lin H, Joo S, Goodenough U: Early Sexual Origins of Homeoprotein Heterodimerization and Evolution of the Plant KNOX/ BELL Family. Cell 2008, 133(5):829-840.

9. Barton MK, Poethig RS: Formation of the shoot apical meristem in Arabidopsis thaliana: an analysis of development in the wild type and in the shoot meristemless mutant. Development 1993, 119(3):823-831.
10. Long JA, Moan El, Medford Jl, Barton MK: A member of the KNOTTED class of homeodomain proteins encoded by the STM gene of Arabidopsis. Nature 1996, 379(6560):66-69.

11. Smith HMS, Campbell BC, Hake S: Competence to Respond to Floral Inductive Signals Requires the Homeobox Genes PENNYWISE and POUND-FOOLISH. Curr Biol 2004, 14(9):812-817.

12. Ragni L, Belles-Boix E, Gunl M, Pautot V: Interaction of KNAT6 and KNAT2 with BREVIPEDICELLUS and PENNYWISE in Arabidopsis Inflorescences. Plant Cell 2008, 20(4):888-900.

13. Byrne ME, Simorowski J, Martienssen RA: ASYMMETRIC LEAVES1 reveals knox gene redundancy in Arabidopsis. Development 2002, 129(8):1957-1965.

14. Reddy GV, Heisler MG, Ehrhardt DW, Meyerowitz EM: Real-time lineage analysis reveals oriented cell divisions associated with morphogenesis at the shoot apex of Arabidopsis thaliana. Development 2004, 131(17):4225-4237.

15. Hamant $O$, Heisler MG, Jonsson $H$, Krupinski $P$, Uyttewaal M, Bokov $P$, Corson F, Sahlin P, Boudaoud A, Meyerowitz EM, et al: Developmental Patterning by Mechanical Signals in Arabidopsis. Science 2008, 322(5908):1650-1655.

16. Fleming AJ, McQueen-Mason S, Mandel T, Kuhlemeier C: Induction of Leaf Primordia by the Cell Wall Protein Expansin. Science 1997, 276(5317):1415-1418.

17. Hernandez LF, Green PB: Transductions for the Expression of Structural Pattern: Analysis in Sunflower. Plant Cell 1993, 5(12):1725-1738.

18. Peaucelle A, Louvet R, Johansen JN, Höfte H, Laufs P, Pelloux J, Mouille G: Arabidopsis Phyllotaxis Is Controlled by the Methyl-Esterification Status of Cell-Wall Pectins. Curr Biol 2008, 18(24):1943-1948.

19. Peaucelle $A$, Louvet $R$, Johansen JN, Salsac F, Morin H, Fournet F, Belcram K, Gillet F, Höfte $H$, Laufs $P$, et al: The transcription factor BELLRINGER modulates phyllotaxis by regulating the expression of a pectin methylesterase in Arabidopsis. Development 2011, 138(21):4733-4741.

20. Sieburth LE, Deyholos MK: Vascular development: the long and winding road. Curr Opin Plant Biol 2006, 9(1):48-54.

21. Cartwright HN, Humphries JA, Smith LG: PAN1: A Receptor-Like Protein That Promotes Polarization of an Asymmetric Cell Division in Maize. Science 2009, 323(5914):649-651.

22. Douglas SJ, Chuck G, Dengler RE, Pelecanda L, Riggs CD: KNAT1 and ERECTA regulate inflorescence architecture in Arabidopsis. Plant Cell 2002, 14(3):547-558

23. Taiz L: Plant-cell expansion - Regulation of cell-wall mechanicalproperties. Annu Rev Plant Physiol Plant Mol Biol 1984, 35:585-657.

24. Emery JF, Floyd SK, Alvarez J, Eshed Y, Hawker NP, Izhaki A, Baum SF, Bowman JL: Radial Patterning of Arabidopsis Shoots by Class III HD-ZIP and KANADI Genes. Curr Biol 2003, 13(20):1768-1774.

25. Zhong $\mathrm{R}$, Ye Z-H: IFL1, a Gene Regulating Interfascicular Fiber Differentiation in Arabidopsis, Encodes a Homeodomain-Leucine Zipper Protein. Plant Cell 1999, 11(11):2139-2152.

26. Frugis G, Giannino D, Mele G, Nicolodi C, Chiappetta A, Bitonti MB, Innocenti AM, Dewitte W, Van Onckelen H, Mariotti D: Overexpression of KNAT1 in lettuce shifts leaf determinate growth to a shoot-like indeterminate growth associated with an accumulation of isopentenyl-type cytokinins. Plant Physio/ 2001, 126(4):1370-1380.

27. Ori N, Eshed Y, Chuck G, Bowman JL, Hake S: Mechanisms that control knox gene expression in the Arabidopsis shoot. Development 2000, 127(24):5523-5532

28. Lincoln C, Long J, Yamaguchi J, Serikawa K, Hake S: A Knotted1-Like Homeobox Gene in Arabidopsis Is Expressed in the Vegetative Meristem and Dramatically Alters Leaf Morphology When Overexpressed in Transgenic Plants. Plant Cell 1994, 6(12):1859-1876.

29. Boyes DC, Zayed AM, Ascenzi R, McCaskill AJ, Hoffman NE, Davis KR, Gorlach J: Growth Stage-Based Phenotypic Analysis of Arabidopsis: A Model for High Throughput Functional Genomics in Plants. Plant Cell 2001, 13(7):1499-1510.

30. Tusher VG, Tibshirani R, Chu G: Significance analysis of microarrays applied to the ionizing radiation response. Proc Natl Acad Sci USA 2001, 98(9):5116-5121.

31. Nieuwland J, Feron R, Huisman BAH, Fasolino A, Hilbers CW, Derksen J, Mariani C: Lipid Transfer Proteins Enhance Cell Wall Extension in Tobacco. Plant Cell 2005, 17(7):2009-2019. 
32. Passardi F, Penel C, Dunand C: Performing the paradoxical: how plant peroxidases modify the cell wall. Trends Plant Sci 2004, 9(11):534-540.

33. Imoto K, Yokoyama R, Nishitani K: Comprehensive approach to genes involved in cell wall modifications in Arabidopsis thaliana. Plant Mol Biol 2005, 58(2):177-192.

34. Grenier J, Potvin C, Trudel J, Asselin A: Some thaumatin-like proteins hydrolyse polymeric $\beta-1,3$-glucans. Plant J 1999, 19(4):473-480.

35. Cole M, Nolte C, Werr W: Nuclear import of the transcription factor SHOOT MERISTEMLESS depends on heterodimerization with BLH proteins expressed in discrete sub-domains of the shoot apical meristem of Arabidopsis thaliana. Nucleic Acids Res 2006, 34(4):1281-1292.

36. Craft J, Samalova M, Baroux C, Townley H, Martinez A, Jepson I, Tsiantis M, Moore I: New pOp/LhG4 vectors for stringent glucocorticoid-dependent transgene expression in Arabidopsis. Plant J 2005, 41(6):899-918.

37. Castle ES: Membrane tension and orientation of structure in the plant cell wall. J Cell Comp Physiol 1937, 10(1):113-121.

38. Wasteneys GO: Progress in understanding the role of microtubules in plant cells. Curr Opin Plant Biol 2004, 7(6):651-660.

39. Corson F, Hamant O, Bohn S, Traas J, Boudaoud A, Couder Y: Turning a plant tissue into a living cell froth through isotropic growth. Proc Nat Acad Sci USA 2009, 106(21):8453-8458.

40. Groover A, Mansfield S, DiFazio S, Dupper G, Fontana J, Millar R, Wang Y: The Populus homeobox gene ARBORKNOX1 reveals overlapping mechanisms regulating the shoot apical meristem and the vascular cambium. Plant Mol Biol 2006, 61(6):917-932.

41. Mele G, Ori N, Sato Y, Hake S: The knotted1-like homeobox gene BREVIPEDICELLUS regulates cell differentiation by modulating metabolic pathways. Genes Dev 2003, 17(17):2088-2093.

42. Venglat SP, Dumonceaux T, Rozwadowski K, Parnell L, Babic V, Keller W, Martienssen R, Selvaraj G, Datla R: The homeobox gene BREVIPEDICELLUS is a key regulator of inflorescence architecture in Arabidopsis. Proc Natl Acad Sci USA 2002, 99(7):4730-4735.

43. Douglas SJ, Riggs CD: Pedicel development in Arabidopsis thaliana: Contribution of vascular positioning and the role of the BREVIPEDICELLUS and ERECTA genes. Dev Biol 2005, 284(2):451-463.

44. Zhong R, Taylor JJ, Ye ZH: Disruption of Interfascicular Fiber Differentiation in an Arabidopsis Mutant. Plant Cell 1997, 9(12):2159-2170.

45. Prigge MJ, Otsuga D, Alonso JM, Ecker JR, Drews GN, Clark SE: Class III homeodomain-leucine zipper gene family members have overlapping, antagonistic, and distinct roles in Arabidopsis development. Plant Cell 2005, 17(1):61-76.

46. Otsuga D, DeGuzman B, Prigge MJ, Drews GN, Clark SE: REVOLUTA regulates meristem initiation at lateral positions. Plant J 2001, 25:223-236.

47. Robischon M, Du J, Miura E, Groover A: The Populus Class III HD ZIP, popREVOLUTA, Influences Cambium Initiation and Patterning of Woody Stems. Plant Physiol 2011, 155(3):1214-1225.

48. Kauschmann A, Jessop A, Koncz C, Szekeres M, Willmitzer L, Altmann T: Genetic evidence for an essential role of brassinosteroids in plant development. Plant J 1996, 9(5):701-713.

49. Mizukami Y, Fischer RL: Plant organ size control: AINTEGUMENTA regulates growth and cell numbers during organogenesis. Proc Natl Acad Sci USA 2000, 97(2):942-947.

50. Kim G-T, Shoda K, Tsuge T, Cho K-H, Uchimiya H, Yokoyama R, Nishitani K, Tsukaya H: The ANGUSTIFOLIA gene of Arabidopsis, a plant CtBP gene, regulates leaf-cell expansion, the arrangement of cortical microtubules in leaf cells and expression of a gene involved in cell-wall formation. EMBO J 2002, 21(6):1267-1279.

51. Vlieghe K, Vuylsteke M, Florquin K, Rombauts S, Maes S, Ormenese S, Van Hummelen $P$, Van de Peer $Y$, Inze D, De Veylder L: Microarray analysis of E2Fa-DPa-overexpressing plants uncovers a cross-talking genetic network between DNA replication and nitrogen assimilation. J Cell Sci 2003, 116(20):4249-4259.

52. Ramirez-Parra E, Lopez-Matas MA, Frundt C, Gutierrez C: Role of an Atypical E2F Transcription Factor in the Control of Arabidopsis Cell Growth and Differentiation. Plant Cell 2004, 16(9):2350-2363.

53. Matsui K, Hiratsu K, Koyama T, Tanaka H, Ohme-Takagi M: A Chimeric AtMYB23 Repressor Induces Hairy Roots, Elongation of Leaves and Stems, and Inhibition of the Deposition of Mucilage on Seed Coats in Arabidopsis. Plant Cell Physiol 2005, 46(1):147-155.

54. Verica JA, Medford Jl: Modified MERI5 expression alters cell expansion in transgenic Arabidopsis plants. Plant Sci 1997, 125(2):201-210.
55. Gleave AP: A versatile binary vector system with a T-DNA organisational structure conducive to efficient integration of cloned DNA into the plant genome. Plant Mol Biol 1992, 20(6):1203-1207.

56. Clough SJ, Bent AF: Floral dip: a simplified method for Agrobacterium-mediated transformation of Arabidopsis thaliana. Plant J 1998, 16(6):735-743.

57. Stears RL, Getts RC, Gullans SR: A novel, sensitive detection system for high-density microarrays using dendrimer technology. Physiol Genomics 2000, 3(2):93-99.

58. Hardstedt M, Finnegan CP, Kirchhof N, Hyland KA, Wijkstrom M, Murtaugh $M P$, Hering BJ: Post-transplant upregulation of chemokine messenger RNA in non-human primate recipients of intraportal pig islet xenografts. Xenotransplantation 2005, 12(4):293-302.

59. Pinon V, Etchells JP, Rossignol P, Collier SA, Arroyo JM, Martienssen RA, Byrne ME: Three PIGGYBACK genes that specifically influence leaf patterning encode ribosomal proteins. Development 2008, 135(7):1315-1324

doi:10.1186/1471-2229-12-212

Cite this article as: Etchells et al: A role for BELLRINGER in cell wall development is supported by loss-of-function phenotypes. BMC Plant Biology 2012 12:212.

\section{Submit your next manuscript to BioMed Central and take full advantage of:}

- Convenient online submission

- Thorough peer review

- No space constraints or color figure charges

- Immediate publication on acceptance

- Inclusion in PubMed, CAS, Scopus and Google Scholar

- Research which is freely available for redistribution

Submit your manuscript at www.biomedcentral.com/submit
C) Biomed Central 